November 15, $2018 \quad$ 9:19 WSPC/INSTRUCTION FILE Coul’sing subm

\title{
THREE-BODY COULOMB FINAL-STATE INTERACTION EFFECTS IN THE COULOMB BREAKUP OF LIGHT NUCLEI
}

\author{
E. O. ALT \\ Institut für Physik, Universität Mainz, D-55099 Mainz, Germany \\ Erwin.Alt@uni-mainz.de \\ B. F. IRGAZIEV \\ Theoretical Physics Department, National University, Tashkent 700174, Uzbekistan \\ qcd@uzsci.net \\ A. M. MUKHAMEDZHANOV \\ Cyclotron Institute, Texas A\&M University, College Station, TX 77843, USA \\ akram@comp.tamu.edu
}

Preprint MZ-TH/05-06

\begin{abstract}
Coulomb breakup of a projectile in the Coulomb field of a fully stripped heavy nucleus is at present one of the most popular experimental methods to obtain information on reactions of interest in nuclear astrophysics. Its theoretical interpretation presents, however, considerable difficulties, due to the three-body nature and the infinite range of the Coulomb forces involved. Among the uncertainties affecting present analyses, the possible modification of the dissociation cross section by three-body Coulomb final-state interactions plays a major role. Various methods which have been proposed to deal with it are briefly reviewed. However, none of them is based on a consistent and mathematically satisfactory quantum mechanical treatment, with the exception of the one proposed recently. The latter, being based on the prior form of the dissociation amplitude, makes use of a genuine three-charged particle wave function for the final state which is an exact solution of the three-body Schrödinger equation, albeit only asymptotically, i.e., for large distances. Nevertheless, interesting conclusion can be drawn concerning the influence of three-body Coulomb final-state interactions on quantities of astrophysical interest.
\end{abstract}

Keywords: THREE-BODY COULOMB BREAKUP; THREE-BODY COULOMB FINAL STATE INTERACTION; NUCLEAR ASTROPHYSICS

PACS Nos: 25.70.Hi, 25.70.Bc, 24.10.Eq, 24.10.Ht

\section{Introduction}

Coulomb breakup reactions, being three- (or more-) problems, are dynamically very complicated due to the influence of strong interactions acting between the colliding nuclei; and the long-range character of the Coulomb interaction adds non-trivially to the complication. However, in the special case of very peripheral collisions the nuclei do not come close to each other so that the short-range nuclear forces between them 
can be neglected. Then the reaction mechanism simplifies considerably: the nuclei interact with each other through the electromagnetic field only and unambiguous conclusions can be drawn from such experiments. Consider the dissociation

$$
a+A \rightarrow b+c+A,
$$

where particle $a$ is the bound state $a=(b c)$, and $A$ is a heavy target. The basic mechanism underlying Coulomb breakup is as follows: the fast projectile $a$ moves in the Coulomb field of the target $A$, with the impact parameter being larger that the sum of the radii of the nuclei $a$ and $A$. The electromagnetic target field then lifts the fragments $b$ and $c$ into the continuum. There they interact with each other via the sum of the Coulomb plus nuclear potentials, but with the target only Coulombically.

Thus, by choosing appropriate kinematic conditions Coulomb breakup represents a powerful, though indirect, tool to obtain information on the radiative capture cross section $b+c \rightarrow a+\gamma$, at astrophysically relevant energies 1 (we also recommend the reader to consult Ref. 2 where the general theory of Coulomb excitation and dissociation and their applications are reviewed). Its advantages are exploited around the world in different nuclear centers (GSI, KfK, Germany, ${ }^{3 / 4}$ GANIL, France, ${ }^{[5}$ NSCL, MSU, Cyclotron Institute, Texas A\&M University, USA, 6789 RIKEN, Japan!10, It is, however, clearly impossible to cover in a short review all experiments where Coulomb breakup reactions have been used to obtain astrophysical information.

Theoretically, Coulomb breakup reactions are studied, e.g., by post and prior forms of the Distorted-Wave Born Approximation (DWBA),111213 the semiclassical approach, 14 the diffractive dissociation approach, 15 and the continuumdiscretized coupled channel (CDCC) method, 161718 each of which has specific advantages and disadvantages. It must, however, be kept in mind that the astrophysical factors ("S-factors") extracted from the analysis of Coulomb breakup data are affected by various uncertainties due to (i) the unavoidable extrapolation of the data to astrophysically effective energies, (ii) contributions from various electromagnetic multipoles $(E 1, E 2, M 1)$, (iii) assumptions about the nuclear interactions, and (iv) various higher-order effects (see Refs. 19202112223 and references therein).

One of the most interesting higher-order effects is the so-called post-decay acceleration (PDA) of the fragments in the Coulomb field of the target. It results from the fact that, if the fragments of the projectile have different charge-over-mass ratios, the repulsive force exerted by the target nucleus will give rise to a different acceleration of each fragment (PDA) which eventually leads to a change in their relative energy on their way from the breakup point to the detector. Consequently, the experimentally accessible amplitude of the projectile photodissociation will differ from the physically interesting one. One of the first direct measurements 9 of the PDA has been performed for the Coulomb breakup ${ }^{7} \mathrm{Li} \rightarrow \alpha+t$ on ${ }^{208} \mathrm{~Pb}$, at a projectile incident energy $E_{\mathrm{i}}=63 \mathrm{MeV}$. There it has been been found that the minimum of the coincidence spectrum of $\alpha$-particles and tritons which is expected to occur at zero relative energy $E_{\alpha t}$, was shifted due to the different acceleration of each fragment in the Coulomb field of the target. Evidently, this different PDA of 
the $\alpha$ and the triton is due to their different charge-over-mass ratios. The shift of the minimum in the coincidence spectrum can serve as an nuclear clock.24

Theoretically the PDA effect has been studied by several authors. The sudden approximation, which takes into account the interaction to all orders but is valid only if the time ordering can be neglected, provides a simple method to determine the higher-order effects. Calculations 14 show that cross sections tend to be smaller than those obtained in first- and second-order perturbation theory, at very small excitation energy which is the region of interest for nuclear astrophysics. A more advanced approach which also takes into account the interaction to all orders is provided by solving directly the time-dependent Schrödinger equation (Refs. 2022252627 and references therein). Here, the motion of the projectile and of the fragments in the Coulomb field of a heavy target are treated quasi-classically assuming straight-line trajectories, but the relative motion of the fragments quantummechanically. However, the time evolution in a Coulomb field does not proceed along a straight line even for large times because of an additional, logarithmically increasing time dependence (which is easily seen by integrating the classical equation of motion in a Coulomb potential). A recent check 28 of the assumption that the projectile and the target do not overlap during the collision ("far-field approximation") has shown that it decreases the $S$-factor extracted from the data of Ref. $\frac{8}{b y} 5 \%$. However, it is not clear how accurate the quasiclassical approach is in the region where the projectile and the target do overlap.

The CDCC method has also been applied! ${ }^{17 / 18}$ As usual the effects of the E2 transition and higher order excitations are included. Since the relative motion of projectile and target, with the huge number of partial waves involved, is treated quantum-mechanically, such kind of approach becomes numerically very involved. In some cases 29 the semiclassical approximation was seen to compare well with corresponding fully quantal calculations at sub-barrier energies. This represents a chance for an enormous simplification in the numerical work since it allows to check and to possibly supersede the CDCC calculations. At higher energies, where also nuclear (grazing) collisions can become important, a Glauber approach is very useful ${ }^{30}$ Alternatively, in the stationary framework use has been made of, among others, the DWBA ${ }^{31}$ and of the quasi-classical approach! ${ }^{32}$ However, in these investigations the three-body scattering wave function for the initial (or final) state is approximated by a product of two two-body or quasi-two-body wave functions which, as will be explained below, actually completely eliminates PDA. For instance, in Refs. 3132 a "local" momentum is introduced which should allow the incorporation of PDA; but in reality it is a "global" momentum because the distance between the spectator and the other two particles which are close to each other is rather arbitrarily fixed at a global value of $10 \mathrm{fm}$. Besides, the method used provokes further questions since the direction of the introduced "local" momentum is undetermined.

In a systematic approach, the dissociation amplitude should be calculated by taking into account the coupling of the different channels, utilizing, for instance, the FRESCO code ${ }^{833}$ However, being presently interested only in an estimation of the 
possible effects of three-body Coulomb final-state interactions which are responsible for PDA on the breakup cross section at small scattering angles and relatively high energies, we take resort to some approximations which are admissible under such conditions. Starting from the prior form of the dissociation amplitude we introduce for the exact three-charged particle wave function occurring therein an approximation which, while simplifying the calculations, still preserves the genuine three-body nature. Thus, the PDA effect will be accounted for in a systematic manner.

The plan of the paper is as follows. Section 2 contains a brief discussion why Coulomb breakup experiments are useful for obtaining information on capture reactions, and a short summary of pertaining experiments and their astrophysical relevance. We then introduce in Sec. 3 the prior-form dissociation amplitude and the (approximate) wave function used to describe the three-charged particle final state. There we also point out that for an investigation of such breakup processes the post form is inappropriate for the derivation of physically correct DWBA or similar approximations. In Sec. 4 some cross section results for the Coulomb breakup reactions ${ }^{6} \mathrm{Li}+{ }^{208} \mathrm{~Pb} \longrightarrow \alpha+d+{ }^{208} \mathrm{~Pb}$ and ${ }^{8} \mathrm{~B}+{ }^{208} \mathrm{~Pb} \longrightarrow{ }^{7} \mathrm{Be}+p+{ }^{208} \mathrm{~Pb}$ are presented from which the impact of PDA on the astrophysical $S$-factor as determined from this reaction can be estimated. Finally, Sec. 5 contains some conclusions.

We use the units in which $\hbar=c=1$. Moreover, unit vectors are denoted by a hat, e. g., $\hat{\mathbf{x}}=\mathbf{x} / x$.

\section{Coulomb breakup reactions and their relation to problems of nuclear astrophysics}

In nuclear astrophysics, radiative capture reactions of the type

$$
b+c \rightarrow a+\gamma
$$

play a very important role. They can also be studied by the time-reversed reaction

$$
\gamma+a \rightarrow b+c
$$

at least in those cases where the nucleus $a$ is in its ground state. The cross sections of reactions (21) and (3) are related by detailed balance,

$$
\sigma(b+c \rightarrow a+\gamma)=\frac{2\left(2 j_{a}+1\right)}{\left(2 j_{b}+1\right)\left(2 j_{c}+1\right)} \frac{k_{\gamma}^{2}}{k_{b c}^{2}} \sigma(\gamma+a \rightarrow b+c) .
$$

The relative momentum between particles $b$ and $c$ is given by $k_{b c}=\sqrt{2 \mu_{b c} E_{b c}}$, with $\mu_{b c}$ being the appropriate reduced mass; the photon momentum is $k_{\gamma}=\left(E_{b c}+Q\right)$, where $Q$ is the $Q$-value of the capture reaction (2). Typically one has $k_{\gamma} / k_{b c} \ll 1$ for energies not in immediate neighborhood of the threshold region $k_{b c} \approx 0$. Due to this phase space factor the cross section for the capture reaction (2) is strongly suppressed in comparison to that of the dissociation reaction (3).

In non-resonant charged particle reactions the energy dependence of the cross section for reaction (2) is dominated by the penetration of the Coulomb barrier. This 
energy dependence is usually factored out by defining the astrophysical $S$-factor

$$
S\left(E_{b c}\right)=E_{b c} \sigma(b+c \rightarrow a+\gamma) \exp (2 \pi \eta),
$$

where $\eta=Z_{b} Z_{c} e^{2} \mu_{b c} / k_{b c}$ is the Coulomb parameter. It is the most characteristic and important quantity for nuclear astrophysics.

In fast peripheral collisions of light nuclei with a heavy nucleus the Coulomb field of the target nucleus creates the equivalent photon spectrum which allows the extraction of the cross section of reaction (2) from Coulomb breakup reactions. ${ }^{1}$ Recent developments, especially of radioactive beams, often permit one to obtain equivalent information with higher-energy beams. The high-energy Coulomb breakup experiments generally yield higher event rates; they also yield sometimes information which is not available in the direct radiative capture reactions.

The ${ }^{6} \mathrm{Li}$ Coulomb dissociation into $\alpha+d$ has been a test case of the Coulomb breakup method. ${ }^{434}$ The experiments were carried out with a $156 \mathrm{MeV}{ }^{6} \mathrm{Li}$ beam in Karlsruhe ${ }^{4}$, and with a $60 \mathrm{MeV}$ beam in Heidelberg!35 This reaction is of astrophysical importance since the $d(\alpha, \gamma)^{6} \mathrm{Li}$ radiative capture is the only process by which ${ }^{6} \mathrm{Li}$ is produced in the standard primordial nucleosynthesis models.

The radiative capture reaction $t(\alpha, \gamma)^{7} \mathrm{Li}$ is astrophysically relevant for the production of the nuclide ${ }^{7} \mathrm{Li}$ in the early universe and during the nucleosynthesis in stars. The most accurate direct capture measurement has been performed in Ref. 36. The Coulomb dissociation method to investigate the ${ }^{7} \mathrm{Li} \rightarrow \alpha+t$ has been applied, too, under various conditions. We mention here the direct breakup of ${ }^{7} \mathrm{Li}$ scattered at $70 \mathrm{MeV}$ off a ${ }^{120} \mathrm{Sn}$ target, 37 at $63 \mathrm{MeV}$ off ${ }^{58} \mathrm{Ni},{ }^{120} \mathrm{Sn},{ }^{144} \mathrm{Sm}$, and ${ }^{208} \mathrm{~Pb}$ targets,, 9 and at $54 \mathrm{MeV}$ off ${ }^{197} \mathrm{Au} \cdot \frac{38}{} \mathrm{~A}$ rather detailed measurement was performed recently, with improved experimental techniques $\underline{39}$

The reaction ${ }^{9} \mathrm{Li} \rightarrow{ }^{8} \mathrm{Li}+n$ was studied with the Coulomb dissociation of a ${ }^{9} \mathrm{Li}$ beam of $28.53 A \cdot \mathrm{MeV}$ at $\mathrm{MSU}{ }^{40}$ to get information on the cross section for the radiative capture reaction ${ }^{8} \mathrm{Li}(n, \gamma){ }^{9} \mathrm{Li}$, which is of importance for the nucleosynthesis in inhomogeneous big bang models and in Type II supernovae.241 $\mathrm{It}$ is also important for determining the different primordial abundances of $\mathrm{Li}, \mathrm{Be}$, $\mathrm{B}$, and $\mathrm{C}$, in order to limit the degrees of freedom in inhomogeneous big bang nucleosynthesis.241

The ${ }^{14} \mathrm{C}(n, \gamma){ }^{15} \mathrm{C}$ reaction plays a role in neutron-induced $\mathrm{CNO}$ cycles of stellar evolution phases beyond the main sequence. It is also involved in the chain of reactions in primordial nucleosynthesis in the neutron-rich environment of an inhomogeneous big bang. Therefore, at MSU a beam of ${ }^{15} \mathrm{C}$ ions of $E_{\mathrm{i}}=35 \mathrm{~A} \cdot \mathrm{MeV}$ was used to study the ${ }^{15} \mathrm{C} \rightarrow{ }^{14} \mathrm{C}+n$ breakup on $\mathrm{C}, \mathrm{Al}, \mathrm{Zn}, \mathrm{Sn}$, and $\mathrm{Pb}$ targets $\frac{42}{2}$

The Coulomb breakup of ${ }^{14} \mathrm{O}$ was measured at RIKEN 4344 and GANIL ${ }^{45}$ in order to determine the $S$-factor of the ${ }^{13} \mathrm{~N}(p, \gamma){ }^{14} \mathrm{O}$ capture reaction which is of astrophysical relevance in the hot $\mathrm{CNO}$ cycle.

The reactions ${ }^{11} \mathrm{C}(p, \gamma){ }^{12} \mathrm{~N}$ which plays a role in the hot $p p$-chain, and ${ }^{22} \mathrm{Mg}(p, \gamma){ }^{23} \mathrm{Al}$ which is relevant for the nucleosynthesis of ${ }^{22} \mathrm{Na}$ in Ne-rich novae, were studied at RIKEN by the Coulomb dissociation method 164748 Their 
result 4647 are consistent with the GANIL measurement. $[5]$ The accuracy of the value for the radiative width of the $1.19 \mathrm{MeV}$ level in ${ }^{12} \mathrm{~N}$ was improved.

Finally, the ${ }^{7} \mathrm{Be}(\mathrm{p}, \gamma)^{8} \mathrm{~B}$ radiative capture reaction is important for the solar neutrino problem which has been solved recently after 40 years of discussions and measurements by many groups. It determines the production rate of ${ }^{8} \mathrm{~B}$ which is the source of high-energy electron neutrinos $\nu_{e}$. In fact, the astrophysical $S$-factor for this reaction must be known to $\pm 5 \%$ in order that this uncertainty not be the dominant error in predictions of the solar $\nu_{e}$ flux. The Kamiokande 49 and $\mathrm{SNO} 50151$ measurements of the neutrino flux from the sun confirmed the standard solar model (cf. Ref. ${ }^{52}$ ). Also the Coulomb dissociation ${ }^{8} \mathrm{~B} \rightarrow{ }^{7} \mathrm{Be}+p$ has been studied at different radioactive beam facilities under different kinematic conditions and energies $(46.5,51.9,83,90$, and $254 \mathrm{~A} \cdot \mathrm{MeV})$ at RIKEN $, 5354 \mathrm{MSU}, \frac{8}{8}$ and GSI $[55$

\section{General Coulomb dissociation amplitude}

Since in the present investigation the main emphasis lies on the elaboration of possible effects of three-body Coulomb final-state interactions on the breakup cross section at small scattering angles and relatively high energies, we take resort to some approximations which are admissible under such conditions.

As starting point we choose the exact dissociation amplitude for the reaction (11) in the prior form,

$$
M_{\mathrm{if}}^{(\text {prior })}=\left\langle\Psi_{\mathrm{f}}^{(-)}|\Delta V| \varphi_{a} \Phi_{\mathrm{i}}^{(+)}\right\rangle, \Delta V=V_{b A}+V_{c A}-U .
$$

Here, $V_{j A}=V_{j A}^{\mathrm{N}}+V_{j A}^{\mathrm{C}}$ is the interaction potential of particle $j(=b, c)$ with the target particle $A$ containing nuclear $\left(V_{j A}^{\mathrm{N}}\right)$ and Coulombic $\left(V_{j A}^{\mathrm{C}}\right)$ parts. The distorted wave $\Phi_{\mathrm{i}}^{(+)}$in the initial channel is the continuum solution of the two-particle Schrödinger equation for the full optical potential $U=U^{\mathrm{C}}+U^{\mathrm{N}}$ and describes the scattering of particles $a$ and $A$ with relative kinetic energy $E_{\mathrm{i}} ; \varphi_{a}$ is the $a=(b c)$ bound state wave function with binding energy $\varepsilon_{b c}$, and $\Psi_{\mathrm{f}}^{(-)}$the full final-state wave function for three charged particles $b, c$, and $A$ in the continuum, with the total energy

$$
E \equiv E_{\mathrm{f}}+E_{b c} \equiv E_{\mathrm{i}}-\varepsilon_{b c} .
$$

The various kinetic energies are defined as $E_{b c}=k_{b c}^{2} / 2 \mu_{b c}$ and $E_{\mathrm{i}, \mathrm{f}}=q_{\mathrm{i}, \mathrm{f}}^{2} / 2 \mu_{a A} ; \mathbf{k}_{b c}$ is the relative momentum of particles $b$ and $c$ in the exit channel, $\mathbf{q}_{\mathrm{i}, \mathrm{f}}$ the relative momentum of $a$ and $A$ in the entry, and of the center of mass of $(b+c)$ and $A$ in the exit channel, respectively. The reduced mass of particles $i$ and $j$ is denoted by $\mu_{i j}=m_{i} m_{j} /\left(m_{i}+m_{j}\right)$.

We assume that the incident center-of-mass energy $E_{\mathrm{i}}$ of the projectile $a$ is high enough and that the scattering angle $\theta$ of the center of mass of the pair $(b+$ $c$ ) is so small (at given $E_{\mathrm{i}}$ ) that the impact parameter is much larger than the nuclear interaction radius $R_{\mathrm{N}}$ between $a$ and $A$. Such conditions are typical for Coulomb breakup processes. Then all potentials in (6) can be approximated by their 
Coulombic parts, $\Delta V \approx \Delta V^{\mathrm{C}}=V_{b A}^{\mathrm{C}}+V_{c A}^{\mathrm{C}}-U^{\mathrm{C}}$. Note that under such conditions, $\Phi_{\mathrm{i}}^{(+)}$is just a two-body Coulomb scattering wave function for the potential $U^{\mathrm{C}}$.

The dissociation amplitude (6) requires knowledge of the three-charged particle wave function $\Psi_{\mathrm{f}}^{(-)}$which is a solution of the three-body Schrödinger equation with incoming-wave three-body boundary conditions,

$$
\left(E-T_{\mathbf{r}}-T_{\boldsymbol{\rho}}-V_{b c}-V_{b A}-V_{c A}\right) \Psi_{\mathbf{k}_{b c}, \mathbf{q}_{\mathrm{f}}}^{(-)}(\mathbf{r}, \boldsymbol{\rho})=0 .
$$

Here, $\mathbf{r}=\mathbf{r}_{b}-\mathbf{r}_{c}$ and $\boldsymbol{\rho}=\left(m_{b} \mathbf{r}_{c}+m_{c} \mathbf{r}_{b}\right) /\left(m_{b}+m_{c}\right)-\mathbf{r}_{A}$, where $\mathbf{r}_{j}$ is the coordinate vector of particle $j$, while $\mathbf{k}_{b c}$ and $\mathbf{q}_{\mathrm{f}}$ are the momenta canonically conjugated to $\mathbf{r}$ and $\boldsymbol{\rho}$, respectively. Hence, $T_{\mathbf{r}}$ and $T_{\boldsymbol{\rho}}$ are the kinetic energy operators corresponding to the relative motion of $b$ and $c$, and to the relative motion of the center of mass of the pair $(b+c)$ and the target $A$, respectively.

Since such a wave function is neither known and nor numerically calculable at present, physically justifiable approximations are unavoidable. Such can be found by the following consideration. The amplitude (6) contains the overlap of the initial bound state wave function $\varphi_{a}(\mathbf{r})$ for the bound pair $(b c)$ and the three-body $(b+$ $c+A$ ) final-state continuum wave function. The former limits the range of variation of $r$. But the range of $\rho$ is not confined and, hence, extends to infinity. Thus, if $\rho \gg R_{\mathrm{N}}^{\prime}$, where $R_{\mathrm{N}}^{\prime}$ is the larger of the nuclear interaction radii between particles $b$ or $c$ and $A$, it suffices to find solutions of the Schrödinger equation restricted to the asymptotic domain $\Omega_{A}:\left\{r \ll \rho, R_{\mathrm{N}}^{\prime} \ll \rho\right\}$, i.e., where the distance $r$ between the fragments $b$ and $c$ is much smaller than that between their center of mass and $A$.

It is easy to see that in $\Omega_{A}$ the Schrödinger equation (8) reduces to

$$
\left\{E-T_{\mathbf{r}}-T_{\boldsymbol{\rho}}-V_{b c}(r)-U^{\mathrm{C}}(\rho)\right\} \Psi_{\mathbf{k}_{b c}, \mathbf{q}_{\mathrm{f}}}^{(+)}(\mathbf{r}, \boldsymbol{\rho})=O\left(r^{2} / \rho^{2}\right),
$$

since there

$$
V_{b A}\left(\mathbf{r}_{b}\right)+V_{c A}\left(\mathbf{r}_{c}\right) \stackrel{\Omega_{A}}{\approx} V_{b A}^{\mathrm{C}}\left(\mathbf{r}_{b}\right)+V_{c A}^{\mathrm{C}}\left(\mathbf{r}_{c}\right)=U^{\mathrm{C}}(\boldsymbol{\rho})+O\left(r^{2} / \rho^{2}\right),
$$

with

$$
V_{j A}^{\mathrm{C}}\left(\mathbf{r}_{b}\right)=Z_{j} Z_{A} e^{2} /\left|\boldsymbol{\rho}+\varepsilon_{j} \mu_{b c} \mathbf{r} / m_{j}\right|, U^{\mathrm{C}}(\rho)=Z_{a} Z_{A} e^{2} / \rho, j=b, c .
$$

Here, $\varepsilon_{j}=+1(-1)$ for $j=b(c)$, and $Z_{j} e$ is the charge of particle $j$.

Since the Hamiltonian in Eq. (9) separates in the variables $\mathbf{r}$ and $\boldsymbol{\rho}$, the obvious solution is provided by a product of two two-body scattering wave functions which we write for later purposes as

$$
\Psi_{\mathrm{f}}^{(-)}(\mathbf{r}, \boldsymbol{\rho}) \equiv \Psi_{\mathbf{k}_{b c}, \mathbf{q}_{\mathrm{f}}}^{(-)}(\mathbf{r}, \boldsymbol{\rho}) \approx e^{i \mathbf{k}_{b c} \cdot \mathbf{r}+i \mathbf{q}_{\mathrm{f}} \cdot \boldsymbol{\rho}} \tilde{\psi}_{\mathbf{k}_{b c}}^{(-)}(\mathbf{r}) \tilde{\Psi}_{\mathbf{q}_{\mathrm{f}}}^{(-)}(\boldsymbol{\rho}) .
$$

Here, $\psi_{\mathbf{k}_{b c}}^{(-)}(\mathbf{r}) \equiv e^{i \mathbf{k}_{b c} \cdot \mathbf{r}} \tilde{\psi}_{\mathbf{k}_{b c}}^{(-)}(\mathbf{r})$ describes the relative motion of particles $b$ and $c$, interacting via the potential $V_{b c}(r)$; and $\Psi_{\mathbf{q}_{\mathrm{f}}}^{(-)}(\boldsymbol{\rho}) \equiv e^{i \mathbf{q}_{\mathrm{f}} \cdot \boldsymbol{\rho}} \tilde{\Psi}_{\mathbf{q}_{\mathrm{f}}}^{(-)}(\boldsymbol{\rho})$ is the scattering wave function of the center of mass of $(b+c)$ and $A$, interacting via the optical potential $U^{\mathrm{C}}(\rho)$. When inserted in the exact expression (6), this leads to the DWBA amplitude conventionally employed in analyses of Coulomb breakup reactions,

$$
M_{\mathrm{if}}^{\text {DWBA }}=\left\langle\psi_{\mathbf{k}_{b c}}^{(-)} \Psi_{\mathbf{q}_{\mathrm{f}}}^{(-)}\left|\Delta V^{\mathrm{C}}\right| \varphi_{a} \Phi_{\mathrm{i}}^{(+)}\right\rangle .
$$


We mention that frequently a wave function $\Psi_{\mathbf{q}_{\mathrm{f}}}^{(-)}(\boldsymbol{\rho})$ calculated with the full optical potential $U^{\mathrm{C}}(\rho)+U^{\mathrm{N}}(\rho)$ is used here 1113

Approximation (12) which has dramatic consequences is motivated by two arguments: (i) the right-hand side of Eq. (12) is indeed an exact solution of the Schrödinger equation (9) restricted to the domain $\Omega_{A}$ which is relevant for Coulomb breakup processes of interest here, and (ii) its use greatly simplifies the evaluation of the dissociation amplitude (6).

While the second argument obviously applies (see below), the first one is grossly misleading. Namely, it comes as a surprise that the condition that Eq. (9) be satisfied up to $O\left(r^{2} / \rho^{2}\right)$ order does not suffice to provide a unique solution $\underline{[56}$ To remove this non-uniqueness and to select the physically acceptable solution, an additional constraint has to be imposed. Such is provided by the requirement that the correct solution of the Schrödinger equation (92) in $\Omega_{A}$ has to smoothly match with the solution of the exact Schrödinger equation (8) in the asymptotic region $\Omega_{0}$ where all three particles $b, c$, and $A$, are well separated, i.e., $r \rightarrow \infty, \rho \rightarrow \infty$, since $\Omega_{0} \cap \Omega_{A} \neq 0$. The leading term of the asymptotic solution in the asymptotic domain $\Omega_{0}$ is given by the Redmond three-body distorted wave,, 57

$$
\begin{aligned}
\Psi_{\mathbf{k}_{b c}, \mathbf{q}_{\mathrm{f}}}^{(-)}(\mathbf{r}, \boldsymbol{\rho}) & \stackrel{\Omega_{0}}{\approx} e^{i \mathbf{k}_{b c} \cdot \mathbf{r}+i \mathbf{q}_{\mathrm{f}} \cdot \boldsymbol{\rho}} e^{-i \eta_{b c} \ln \zeta_{b c}} e^{-i \eta_{b A} \ln \zeta_{b A}} e^{-i \eta_{c A} \ln \zeta_{c A}} \\
& \stackrel{\Omega_{0}}{\approx} e^{i \mathbf{k}_{b c} \cdot \mathbf{r}+i \mathbf{q}_{\mathrm{f}} \cdot \boldsymbol{\rho}} \tilde{\psi}_{\mathbf{k}_{b c}^{C(-)}}^{C\left(\mathbf{r}_{b c}\right)} \tilde{\psi}_{\mathbf{k}_{b A}}^{C(-)}\left(\mathbf{r}_{b A}\right) \tilde{\psi}_{\mathbf{k}_{c A}}^{C(-)}\left(\mathbf{r}_{c A}\right),
\end{aligned}
$$

where the second form $\frac{58}{18}$ is asymptotically equivalent to the first one. Here, $\eta_{i j}$ is the Coulomb parameter, $\zeta_{i j}=k_{i j} r_{i j}-\mathbf{k}_{i j} \cdot \mathbf{r}_{i j}$, and $\mathbf{r}_{i j}\left(\mathbf{k}_{i j}\right)$ the relative coordinate (relative momentum) between particles $i$ and $j$. The wave functions $\psi_{\mathbf{k}_{i j}}^{C(-)}\left(\mathbf{r}_{i j}\right) \equiv$ $e^{i \mathbf{k}_{i j} \cdot \mathbf{r}_{i j}} \tilde{\psi}_{\mathbf{k}_{i j}}^{C(-)}\left(\mathbf{r}_{i j}\right)$ describe the scattering of particles $i$ and $j$, interacting via the Coulomb potential $V_{i j}^{C}\left(r_{i j}\right)$ (with incoming wave boundary condition).

It is apparent, and in fact a simple exercise to check, that expression (12) does not match in the leading order with the asymptotic solution (14) in $\Omega_{0}$ and, hence, is not admissible. That defect notwithstanding, it is conventionally taken as the approximation for the final-state three-body wave function $\Psi_{\mathrm{f}}^{(-)}$.

Thus, an alternative approximation for $\Psi_{\mathrm{f}}^{(-)}$must be found which, while satisfying the above constraints, nevertheless renders the dissociation amplitude calculable. A three-body wave function which satisfies all these conditions has been derived in Refs. 5659 . The leading order term $[56$ can be written as

$$
\begin{aligned}
\Psi_{\mathrm{f}}^{(-)(\mathrm{as})}(\mathbf{r}, \boldsymbol{\rho}) \stackrel{\Omega_{A}}{\approx} e^{i \mathbf{k}_{b c} \cdot \mathbf{r}+i \mathbf{q}_{\mathrm{f}} \cdot \boldsymbol{\rho}} \tilde{\psi}_{\mathbf{k}_{b c}(\boldsymbol{\rho})}^{(-)}(\mathbf{r}) e^{-i \eta_{b A} \ln \zeta_{b A}} e^{-i \eta_{c A} \ln \zeta_{c A}} \\
\\
\simeq e^{i \mathbf{k}_{b c} \cdot \mathbf{r}+i \mathbf{q}_{\mathrm{f}} \cdot \boldsymbol{\rho}} \tilde{\psi}_{\mathbf{k}_{b c}(\boldsymbol{\rho})}^{(-)}(\mathbf{r}) \tilde{\psi}_{\mathbf{k}_{b A}}^{(-)}\left(\mathbf{r}_{b A}\right) \tilde{\psi}_{\mathbf{k}_{c A}}^{(-)}\left(\mathbf{r}_{c A}\right) .
\end{aligned}
$$

The new wave function $\psi_{\mathbf{k}_{b c}(\boldsymbol{\rho})}^{(-)}(\mathbf{r}) \equiv e^{i \mathbf{k}_{b c}(\boldsymbol{\rho}) \cdot \mathbf{r}_{b c}} \tilde{\psi}_{\mathbf{k}_{b c}(\boldsymbol{\rho})}^{(-)}(\mathbf{r})$ describes the scattering of particles $b$ and $c$, interacting with the full potential $V_{b c}(r)$ and moving with relative momentum $\mathbf{k}_{b c}(\boldsymbol{\rho})$. As a consequence of the long-range Coulombic threebody correlations between $b$ and $A$ and between $c$ and $A$ the latter, however, depends 
on the distance of their center of mass to the charged particle $A$, and is given by

$$
\mathbf{k}_{b c}(\boldsymbol{\rho})=\mathbf{k}_{b c}-\frac{\mathbf{a}(\hat{\boldsymbol{\rho}})}{\rho}, \mathbf{a}(\hat{\boldsymbol{\rho}}):=\mu_{b c}\left[\frac{\eta_{b A}}{m_{b}} \frac{\left(\hat{\mathbf{k}}_{b A}+\hat{\boldsymbol{\rho}}\right)}{\left(1+\hat{\boldsymbol{\rho}} \cdot \hat{\mathbf{k}}_{b A}\right)}-\frac{\eta_{c A}}{m_{c}} \frac{\left(\hat{\mathbf{k}}_{c A}+\hat{\boldsymbol{\rho}}\right)}{\left(1+\hat{\boldsymbol{\rho}} \cdot \hat{\mathbf{k}}_{c A}\right)}\right] .
$$

It is not difficult to see that the wave function (16) has the desired properties: (i) it is asymptotically solution of the asymptotic Schrödinger equation (9) and (ii) it smoothly matches with the Redmond wave function in $\Omega_{0}$ and, hence, is asymptotically also solution of the original Schrödinger equation (8).

It is apparent that the three-charged particle wave function (16) which, as we emphasize once more, is the only physically admissible, asymptotically exact solution of the three-body Schrödinger equation (9) for use in the dissociation amplitude (6), is much more complicated than (the asymptotic part of) the simple factorized form (12) conventionally used in DWBA calculations. Insertion into (6) yields for the appropriate dissociation amplitude

$$
M_{\mathrm{if}}^{(\text {prior })} \approx\left\langle\Psi_{\mathrm{f}}^{(-)(\text {as })}\left|\Delta V^{\mathrm{C}}\right| \varphi_{a} \Phi_{\mathrm{i}}^{(+)}\right\rangle .
$$

Since the integration over $r$ is limited by the bound state wave function $\varphi_{a}(\mathbf{r})$ of the projectile $a$, and if the impact parameter is larger than the nuclear interaction radius $R_{\mathrm{N}}$ between $a$ and $A$, we can utilize the multipole expansion for $\Delta V$. It leads to the multipole expansion of the dissociation amplitude (18):

$$
M_{\mathrm{if}}=\sum_{L M} \frac{4 \pi}{2 L+1} M_{L M}
$$

The multipole transition matrix elements $M_{L M}$ are defined as

$$
\begin{aligned}
M_{L M}= & Z_{A} Z_{L}^{(\mathrm{eff})} e^{2} \int d \boldsymbol{\rho} \int d \mathbf{r} \Psi_{\mathrm{f}}^{(-)(\mathrm{as}) *}(\mathbf{r}, \boldsymbol{\rho}) \\
& \times \frac{r^{L}}{\rho^{L+1}} Y_{L M}^{*}(\mathbf{r}) Y_{L M}(\hat{\boldsymbol{\rho}}) \varphi_{a}(\mathbf{r}) \Phi_{\mathbf{q}_{\mathbf{i}}}^{(+)}(\boldsymbol{\rho}),
\end{aligned}
$$

with $Z_{L}^{(\text {eff })}=\mu_{b c}^{L}\left[(-1)^{L} Z_{b} / m_{b}^{L}+Z_{c} / m_{c}^{L}\right]$ and $\Phi_{\mathrm{i}}^{(+)}(\boldsymbol{\rho}) \equiv \Phi_{\mathbf{q}_{\mathrm{i}}}^{(+)}(\boldsymbol{\rho})$. Note that if in $\Psi_{\mathrm{f}}^{(-)(\text {as })}$ also the next-to-leading-order terms are taken into account, two more terms of similar structure but suppressed by an additional factor $1 / \rho$ occur in (20) $[59$

The right-hand side of Eq. (20) contains a six-dimensional quadrature. This is to be contrasted with the conventional approach. There, the multipole matrix elements of the DWBA amplitude (13) separate into a product of two terms, each requiring three-dimensional quadratures. This greatly reduces the numerical expense.

Use of the amplitudes (18), resp. (20), which are based on the prior-form dissociation amplitude has two crucial consequences. First, since the wave function occurring therein has the correct asymptotic behavior this expression is consistent with the general principles of scattering theory of charged particles. This is to be contrasted with the standard DWBA of the dissociation amplitude (13) the derivation of which usually starts from the post form, $M_{\mathrm{if}}^{(\text {post })}=\left\langle\psi_{b c}^{(-)} \Phi_{a A}^{(-)}|\Delta V| \Psi_{\mathrm{i}}^{(+)}\right\rangle$, with $\Delta V$ as in Eq. (6). The final-state wave function is given by the right-hand side 
of Eq. (12). The exact initial-state three-body wave function is then approximated by the product $\Psi_{\mathrm{i}}^{(+)} \approx \varphi_{a} \Phi_{\mathrm{i}}^{(+)}$. However, in the presence of Coulomb potentials acting between all three particles, the post-form amplitude is not valid, in contrast to the prior form (6), and hence is not equivalent to the latter. The reason is that the final-state product wave function occurring therein does not produce the correct behavior in any asymptotic region of configuration space. Secondly, as mentioned earlier, the PDA effect occurs for $Z_{b} / m_{b} \neq Z_{c} / m_{c}$, since it is caused by the difference in the Coulomb forces exerted by the target on each fragment $b$ and $c$, which leads to the different accelerations. This fact gives rise to a change of the relative velocity of the fragments when they finally have reached the detectors as compared to their relative velocity at the moment of the decay of the projectile $a$. Use of approximation (12), however, completely eliminates the PDA effect because (i) the wave function part describing the scattering of the fragments $b$ and $c$ in the Coulomb field of the target $A$ is approximated by $\Psi_{\mathbf{q}_{\mathrm{f}}}^{(-)}(\boldsymbol{\rho})$ which describes the scattering of the center of mass of the pair $(b+c)$ off the target rather than the scattering of the individual fragments, and, related to it, (ii) the relative motion wave function $\psi_{\mathbf{k}_{b c}}^{(-)}(\mathbf{r})$ of the fragments $b$ and $c$ in the continuum contains the asymptotic relative momentum between $b$ and $c$ and, hence, does not allow for its modification due to the action of the target Coulomb field between the breakup point and the counter.

\section{Applications}

\section{1. ${ }^{6}$ Li breakup into $\alpha$-particle plus deuteron}

As a first application of the proposed method we have computed $\frac{60}{6}$ the tripledifferential cross section (TDCS) for the reaction

$$
{ }^{6} \mathrm{Li}+{ }^{208} \mathrm{~Pb} \longrightarrow \alpha+d+{ }^{208} \mathrm{~Pb},
$$

for a projectile energy $E_{\mathrm{i}}=156 \mathrm{MeV}$ and scattering angles $\theta_{\alpha}=\theta_{d}=3^{\circ}$. In this investigation only the leading term of the asymptotic wave function (16) has been retained. Moreover, because of the peripherality of the reaction it suffices to take into account only the Coulomb interaction between the fragments in the final state so that $\psi_{\mathbf{k}_{\alpha d}(\boldsymbol{\rho})}^{(-)}(\mathbf{r})$ is simply a Coulomb scattering wave function, albeit with local momentum $\mathbf{k}_{\alpha d}(\boldsymbol{\rho})$. Since for $\rho>R_{\mathrm{N}}$, the additional term $\mathbf{a}(\hat{\boldsymbol{\rho}}) / \rho$ in the local momentum turned out to be much smaller in magnitude than the asymptotic momentum $\mathbf{k}_{\alpha d}$, this wave function was expanded in powers of $|\mathbf{a}(\boldsymbol{\rho})| / \rho k_{\alpha d}$. Keeping only the leading terms we arrive at $(\psi(z)$ the digamma function)

$$
\begin{aligned}
\psi_{\mathbf{k}_{\alpha d}(\boldsymbol{\rho})}^{(-)}(\mathbf{r}) & \approx\left[1+\frac{A\left(\hat{\boldsymbol{\rho}}, \mathbf{k}_{\alpha d}\right)}{\rho}\right] \psi_{\mathbf{k}_{\alpha d}}^{C(-)}(\mathbf{r}), \\
A\left(\hat{\boldsymbol{\rho}}, \mathbf{k}_{\alpha d}\right) & =\frac{\mathbf{a}(\hat{\boldsymbol{\rho}}) \cdot \hat{\mathbf{k}}_{\alpha d} \eta_{\alpha d}}{k_{\alpha d}}\left[\frac{\pi}{2}+i \psi\left(1-i \eta_{\alpha d}\right)\right] .
\end{aligned}
$$

Use of (22) greatly simplifies the calculation of the amplitudes $M_{L M}$ since each of them separates into a product of two quantities contaning integration over either $\mathbf{r}$ 
or $\boldsymbol{\rho}$ only. Thus, in this approximation the whole effect of PDA resides in the factor $A\left(\hat{\boldsymbol{\rho}}, \mathbf{k}_{\alpha d}\right)$; or explicitly, for $A\left(\hat{\boldsymbol{\rho}}, \mathbf{k}_{\alpha d}\right)=0$ PDA does not occur.

Both $E 1$ and $E 2$ transitions were included. In the isospin formalism, for the ${ }^{6} \mathrm{Li} \rightarrow \alpha+d$ breakup the $E 1$ transition is forbidden $(\Delta T=0)$; thus the main contribution comes from $E 2$. Nevertheless, since the isospin rule is violated and the charge-over-mass ratio $Z_{\alpha} / m_{\alpha}$ of the $\alpha$-particle differs only little from that of the deuteron, $E 1$ contributes comparably to $E 2$ for $E_{\alpha d}<100 \mathrm{keV}$ (see also Ref. 61 where the contribution of $E 1$ to the reaction $\alpha+d \rightarrow{ }^{6} \mathrm{Li}+\gamma$ is discussed).

In Fig. 1 1 we present the calculated TDCS. Inspection shows that for $\left|E_{\alpha d}\right|<300$ $\mathrm{keV}$ the agreement between the experimenta 4 and theoretical results is very good. Hereby, the notation is such that positive (negative) values of $E_{\alpha d}$ are associated

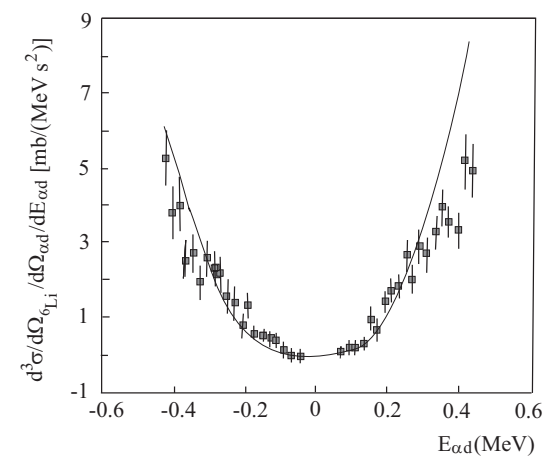

Fig. 1. The TDCS for the reaction ${ }^{6} \mathrm{Li}+{ }^{208} \mathrm{~Pb} \longrightarrow \alpha+d+{ }^{208} \mathrm{~Pb}$ at a projectile energy $E_{\mathrm{i}}=156$ $\mathrm{MeV}$ and scattering angle $\theta=3^{\circ}$ of the fragments, as a function of the $\alpha-d$ relative kinetic energy $E_{\alpha d}$. Negative (positive) values correspond to $\alpha-d$ relative velocities $v_{\alpha d}<0\left(v_{\alpha d}>0\right)$. Experimental data are from Ref. 4 .

with "forward" and "backward" emission of the $\alpha$-particle in the center of mass system of the decaying ${ }^{6} \mathrm{Li}$ nucleus. The astrophysical $S_{24}$-factor for $E_{\alpha d}=0 \mathrm{ex}$ tracted therefrom (by linear extrapolation into the energy range $E_{\alpha d}<20 \mathrm{keV}$ ) is $S_{24}(0)=1.2 \mathrm{nb} \cdot \mathrm{MeV}$. This value agrees with that obtained $\sqrt{62}$ from a calculation of the cross section for the radiative capture $d(\alpha, \gamma)^{6} \mathrm{Li}$ at ultra-low energies $E_{\alpha d}$, but disagrees with the old extrapolated value $S_{24}^{\exp }(0)=9.1 \pm 1.8 \mathrm{nb} \cdot \mathrm{MeV}$ in Ref. 44. Note that an earlier analysis 63 of the same data gave a value of $S_{24}(0)=1.7 \mathrm{nb} \cdot \mathrm{MeV}$.

Fig. 2 illustrates the role of the factor $A\left(\hat{\boldsymbol{\rho}}, \mathbf{k}_{\alpha d}\right)$, which comprises within our approximations all three-body Coulomb final-state interaction effects to the cross section, in the energy region $\left|E_{\alpha d}\right|>20 \mathrm{keV}$ (because of our assumption $\left|A\left(\hat{\boldsymbol{\rho}}, \mathbf{k}_{\alpha d}\right)\right| / \rho \ll 1$ the curves cannot be extrapolated to the point $\left.E_{\alpha d}=0\right)$. Nevertheless it is apparent that the ratio of the cross sections calculated with the conventional asymptotic wave function $\left[A\left(\hat{\boldsymbol{\rho}}, \mathbf{k}_{\alpha d}\right)=0\right]$ to the one calculated with the asymptotically correct wave function, which takes into account three-body Coulomb effects $\left[A\left(\hat{\boldsymbol{\rho}}, \mathbf{k}_{\alpha d}\right) \neq 0\right]$, behaves differently in the regions $v_{\alpha d}<0$ and $v_{\alpha d}>0$. That 


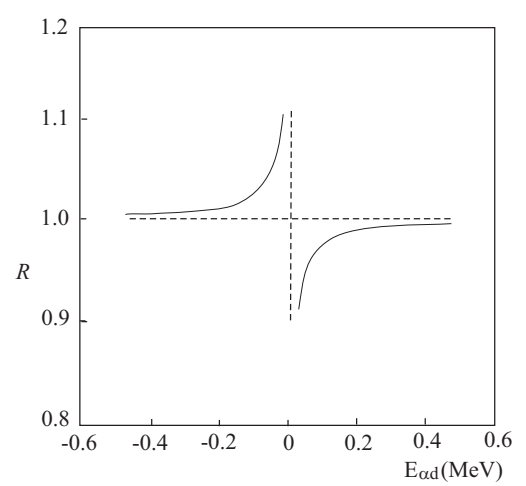

Fig. 2. Ratio $\frac{d^{3} \sigma(A=0) / d \Omega_{6} L_{i} / d \Omega_{\alpha d} / d E_{\alpha d}}{d^{3} \sigma(A \neq 0) / d \Omega_{6} / d \Omega_{\alpha d} / d E_{\alpha d}}$ of the TDCS calculated without $\left(A\left(\hat{\boldsymbol{\rho}}, \mathbf{k}_{\alpha d}\right)=0\right)$ and with $\left(A\left(\hat{\boldsymbol{\rho}}, \mathbf{k}_{\alpha d}\right) \neq 0\right)$ allowance for three-body Coulomb effects, as function of $\alpha-d$ relative kinetic energy $E_{\alpha d}$. Negative and positive values of $E_{\alpha d}$ have the same meaning as in Fig. 1

is, three-body Coulomb effects, together with the interference of E1 and E2 transitions, give rise to an asymmetry of the cross section ratio with respect to the point $v_{\alpha d}=0$. Hence, it is compulsory to accurately take them into account when deducing the $S_{24}$-factor from the TDCS.

\section{2. ${ }^{8} \mathrm{~B}$ breakup into ${ }^{7} \mathrm{Be}$ plus proton}

As a further example we have investigated $\frac{6465}{65}$ the breakup reaction

$$
{ }^{8} \mathrm{~B}+{ }^{208} \mathrm{~Pb} \longrightarrow{ }^{7} \mathrm{Be}+p+{ }^{208} \mathrm{~Pb},
$$

at ${ }^{8} \mathrm{~B}$ beam energies $E_{\mathrm{i}}=46.5 \mathrm{~A} \cdot \mathrm{MeV} \sqrt{53}$ and $E_{\mathrm{i}}=83 \mathrm{~A} \cdot \mathrm{MeV}, \frac{8}{\text { taking into account }}$ dipole and quadrupole contributions. ${ }^{7} \mathrm{Be}-p$ relative kinetic energies are varied between $100 \mathrm{keV}$ and $1 \mathrm{MeV}$. Recall that this reaction allows the extraction of the astrophysical $S$-factor for the important radiative capture reaction ${ }^{7} \mathrm{Be}+p \rightarrow{ }^{8} \mathrm{~B}+\gamma$. The final ${ }^{7} \mathrm{Be}$ nucleus is assumed to be in the ground state. Note that in the kinematic region of interest, both the binding energy $\varepsilon^{\varepsilon_{\mathrm{B}} \mathrm{p} p}=0.137 \mathrm{MeV}$ of the proton to the ${ }^{7} \mathrm{Be}$ nucleus as well as the kinetic energy of the relative motion of the proton and ${ }^{7} \mathrm{Be}$ in the final state are much smaller than the collision energy $E_{\mathrm{i}}$.

When using the asymptotic three-body wave function including next-to-leadingorder terms $\$ 59$ for the final state, the breakup amplitude is given as a sum of three terms. The first one arises from the leading term (18) (of $\left.O\left(\rho^{0}\right)\right)$ and is expected to give the largest contribution. Indeed, it turned out that the cross section is completely governed by it. The other terms are suppressed by an additional factor $1 / \rho$ in the wave function, contributing less than $1 \%$ to the cross section.

The dipole transition to the ${ }^{7} \mathrm{Be}-p$ s-wave continuum state is found to dominate the amplitude; the contribution of the dipole transition to the $d$-wave state is small at small relative kinetic energies $E_{7^{3} \mathrm{Be}}$ but increases up to $30 \%$ at $1 \mathrm{MeV}$. Moreover, in our approach the quadrupole amplitude depends only on the projectile energy 
$E_{\mathrm{i}}$ and on the ${ }^{7}$ Be- $p$ relative energy $E_{{ }^{7} \mathrm{Be} p}$ but not on the scattering angle $\theta$. Its contribution relative to that coming from the dipole amplitude changes from a few per cent at small $E_{7_{B e p}}$ and small $\theta$ to up to $30 \%$ near the grazing angle. We mention that the same behavior of the quadrupole term was observed in a calculation using first- and second-order perturbation theory in the semiclassical approach 14 The consequence is that for radiative capture reactions at energies of interest in nuclear astrophysics, the quadrupole amplitude is negligible. For details we refer to Ref. 65.

Typical results are presented in Tables 1 and 2 Table 1 shows the ratio of the double differential cross section (DDCS), calculated with the (asymptotic) threebody wave function (16), as compared to the one using the conventional ansatz (12) for the final state, at incident energies $E_{\mathrm{i}}=46.5 A \cdot \mathrm{MeV}$. The former takes

Table 1. Ratio $R(\theta)$ of the DDCS for the reaction ${ }^{208} \mathrm{~Pb}\left({ }^{8} \mathrm{~B},{ }^{7} \mathrm{Be} p\right){ }^{208} \mathrm{~Pb}$, calculated with the asymptotic three-body Coulomb wave function [16, to the DDCS calculated using the conventional wave function (12), as a function of the scattering angle $\theta$, for various values of the ${ }^{7} \mathrm{Be}-p$ relative energy. The projectile energy is $E_{\mathrm{i}}=46.5 \mathrm{~A} \cdot \mathrm{MeV}$.

\begin{tabular}{cccccccccccc}
\hline$E_{7_{\mathrm{Be} p}}$ & \multicolumn{10}{c}{$R(\theta)$} \\
\cline { 2 - 13 }$[\mathrm{MeV}]$ & $1.0^{\circ}$ & $1.5^{\circ}$ & $2.0^{\circ}$ & $2.5^{\circ}$ & $3.0^{\circ}$ & $3.5^{\circ}$ & $4.0^{\circ}$ & $4.5^{\circ}$ & $5.0^{\circ}$ & $5.5^{\circ}$ & $6.0^{\circ}$ \\
\hline 0.1 & 1.16 & 1.26 & 1.37 & 1.49 & 1.63 & 1.77 & 1.91 & 2.04 & 2.17 & 2.30 & 2.41 \\
\hline 0.15 & 1.02 & 1.04 & 1.07 & 1.11 & 1.14 & 1.16 & 1.19 & 1.21 & 1.23 & 1.24 & 1.25 \\
\hline 0.2 & 1.01 & 1.01 & 1.02 & 1.03 & 1.03 & 1.03 & 1.03 & 1.03 & 1.02 & 1.02 & 1.01 \\
\hline 0.4 & 1.00 & 1.00 & 0.99 & 0.99 & 0.98 & 0.98 & 0.97 & 0.96 & 0.95 & 0.94 & 0.92 \\
\hline 0.6 & 1.00 & 1.00 & 1.00 & 0.99 & 0.99 & 0.99 & 0.98 & 0.98 & 0.98 & 0.97 & 0.97 \\
\hline 0.8 & 1.00 & 1.00 & 1.00 & 1.00 & 1.00 & 1.00 & 0.99 & 0.99 & 0.99 & 0.99 & 0.99 \\
\hline 1.0 & 1.00 & 1.00 & 1.00 & 1.00 & 1.00 & 1.00 & 1.00 & 1.00 & 1.00 & 1.01 & 1.01 \\
\hline
\end{tabular}

into account three-body Coulomb final state interactions and, hence, PDA while the latter does not. Thus, deviation of this ratio from unity is a direct measure of the influence of PDA. We only mention that a calculation at $83 A \cdot \mathrm{MeV}$ shows that PDA effects decrease with increasing projectile energy and relative energy of the outgoing fragments. But at small relative energies and large scattering angles, PDA is important, the deviation from unity reaching more than $10 \%$. Moreover, from Table 2 we conclude that PDA affects the single differential cross section (SDCS) less than the DDCS. This is understandable because PDA is large near the grazing angle where the cross section is small; and the integration over the scattering angle of the DDCS levels out its contribution. We mention that our approach may produce a large error near the grazing angle where the nuclear interaction between

Table 2. The ratio as in Table 1 but for the SDCS $d \sigma / d E_{7 \mathrm{Be} p}$, at $E_{\mathrm{i}}=46.5$ and $83 A \cdot \mathrm{MeV}$, obtained by integrating the DDCS over the scattering angle $\theta \in\left[2^{\circ}-6^{\circ}\right]$ and $\left[0.1-1.77^{\circ}\right]$, respectively.

\begin{tabular}{lccccccc}
\hline$E_{7_{\text {Bep }}}[\mathrm{MeV}]$ & 0.1 & 0.15 & 0.2 & 0.4 & 0.6 & 0.8 & 1.0 \\
\hline Ratio $\left(E_{\mathrm{i}}=46.5 \mathrm{~A} \cdot \mathrm{MeV}\right)$ & 1.90 & 1.18 & 1.03 & 0.97 & 0.98 & 1.00 & 1.00 \\
Ratio $\left(E_{\mathrm{i}}=83 \mathrm{~A} \cdot \mathrm{MeV}\right)$ & 1.13 & 1.02 & 1.00 & 1.00 & 1.00 & 1.00 & 1.00 \\
\hline
\end{tabular}


the projectile and the target becomes important which has been omitted here.

From these results the following conclusions can be drawn.

(i) At $46.5 \mathrm{~A} \cdot \mathrm{MeV}$ incident energy, the DDCS is enhanced by three-body Coulomb final-state interactions only at the lowest relative energies $E_{7^{B} \mathrm{Be}}$ while in the medium energy range it is reduced; at the highest energies considered their effect becomes negligible. That is, the three-body effects increase the probability of dissociation of ${ }^{8} \mathrm{~B}$ up to energies of $E_{7_{\mathrm{Be}}} \sim 200 \mathrm{keV}$, but start suppressing it at $\sim 400 \mathrm{keV}$.

(ii) Three-body Coulomb effects become more important as $\theta$ increases. The rather small influence at very small $\theta$ is due to the fact that there large impact parameters dominate for which the three-body correlations are weak. Thus, in order to avoid PDA effects, experiments at $E_{\mathrm{i}}=46.5 \mathrm{~A} \cdot \mathrm{MeV}$ should be performed at $\theta \leq 4^{\circ}$.

(iii) On the other hand, PDA effects decrease as the incident energy increases, cf. Table 2 which compares the SDCS ratios with and without three-body effects, at

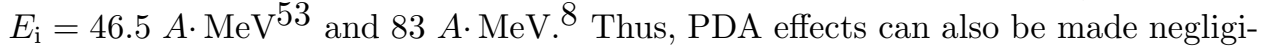
bly small by choosing sufficiently large projectile energies.

(iv) Since our present approach is based on an asymptotic three-particle wave function and, hence, does not allow to perform the calculations for small enough ${ }^{7} \mathrm{Be}-p$ relative kinetic energies, extrapolation to $E_{7^{\mathrm{Be}} p}=0$ seems too arguable. Hence, no reliable quantitative predictions about the influence of three-body Coulomb effects on the Coulomb dissociation cross section and the $S_{17}$-factor to be extracted from it can be made. However, qualitative conclusions, in particular under which kinematic conditions PDA effects are negligible, can already be drawn. This shortcoming is shared also by the standard DWBA approaches based on Eq. (13) since there also only a solution, and indeed an unacceptable one, of the asymptotic Schrödinger equation (9), and not a genuinely calculated three-body wave function is used.

\section{Summary and outlook}

Peripheral collisions of medium- and high-energy nuclei, that is collisions in which these nuclei pass a heavy, fully stripped target nucleus at distances beyond the nuclear interaction radius and which are therefore dominated by electromagnetic interactions, have evolved into an important tool of the nuclear astrophysical research, because of the intense source of quasi-real photons available from the target in such collisions. In addition new experimental facilities, in particular the present and forthcoming radioactive beam factories, render feasible the efficient investigation of photo-interactions with (stable and unstable) nuclei, such as single- and multi-photon excitations and electromagnetic dissociation.

In Coulomb breakup processes, among the various so-called higher-order effects the post-decay acceleration (PDA) of the fragments in the target Coulomb field which is due to three-body Coulomb final-state interactions plays an important role, especially at low and medium collision energies. Various theoretical and computational methods presently in use for taking them into account have been briefly sketched. We have, in particular, emphasized that the frequently used conventional 
separation of the dissociation amplitude into a product of a purely two-body excitation amplitude and a quasi-two-body scattering part of the center of mass of the projectile has the consequence that all PDA effects are eliminated.

To overcome this problem we have proposed an approach which makes use of a genuine three-charged particle wave function to describe the final state. The latter is solution of the three-body Schrödinger equation, albeit only in an asymptotic region of configuration space which, however, is relevant for breakup processes. Because of this our estimation of three-body final-state interaction effects is reliable only for small scattering angles. For larger angles a more accurate three-body Coulomb scattering wave function has to be used which is not yet available. Therefore, further investigations of the PDA effects are called for. Nevertheless, we have shown that already the use of even an only asymptotically correct wave function for three charged particles in the continuum is beneficial for extracting reliable values of astrophysical factors from Coulomb breakup reactions, or at least can help pinpointing at kinematic conditions in which PDA effects should play a negligible role. We finally mention that the discovery of a low lying $E 1$-strength in neutron-rich nuclei and the demand to determine astrophysical $S$-factors of radiative capture processes, as for ${ }^{7} \mathrm{Be}(p, \gamma){ }^{8} \mathrm{~B}$, inspires new calculations, possibly with new computational techniques, of Coulomb breakup processes. Thus, Coulomb dissociation provides comprehensive opportunities to study the interaction of exotic nuclei with photons.

We have not mentioned the Coulomb breakup through excitation of a resonance state of the projectile which is expected to lead to interesting results in experimental as well as theoretical respect.

Among the problems still to be solved are (i) from the experimental side the accurate determination of the relative energy of the fragments, (ii) from the theoretical side the extrapolation of the astrophysical factors to zero relative energy, the accurate determination of the $E 2$ contribution, relativistic corrections and the nuclear interference terms in the cross section which become more and more important as the scattering angle increases, especially if it approaches the grazing angle.

\section{Acknowledgments}

This work was supported by the U. S. DOE under Grant No. DE-FG03-93ER40773, by NSF Award No. PHY-0140343, by Deutscher Akademischer Austauschdienst (DAAD) and by the Center of Sciences and Technology of Uzbekistan, Project No \$2.1.44. B. F. I. would like to thank the Cyclotron Institute of Texas A\&M University and the Institut für Physik der Universität Mainz for the hospitality extended to him during his visits.

\section{References}

1. G. Baur, C. A. Bertulani, and H. Rebel, Nucl. Phys. A458, 188 (1986).

2. G. Baur, K. Hencken, D. Trautmann, Prog. Part. Nucl. Phys. 51, 487 (2003) (nucl-th/0304041). 
3. H. H. Gutbrod et al. (Eds), An International Accelerator Facility for Beams of Ions and antiprotons, GSI, Darmstadt (2001); see website at http://www gsi. de/ .

4. J. Kiener et al., Phys. Rev. C 44, 2195 (1991).

5. A. Lefebvre et al., Nucl. Phys. A592, 69 (1995).

6. NSCL, Scientific Opportunities with Fast Fragmentation Beams from RIA; see http://www.nscl.msu.edu/ria/process/whitepapers/opportunitiesffbeam.pdf (2000).

7. National Superconducting Cylcotron Laboratory, Workshops and White Papers, see webpage at http://www nscl. msu. edu/ria/process/index. php/whitepapers/.

8. B. Davids et al., Phys. Rev. C 63, 065806 (2001).

9. H. Utsunomiya et al., Nucl. Phys. A 511, 379 (1990).

10. RIKEN RI Beam Factory, see webpage at http://www rarf. riken. go. jp/.

11. G. Baur, F. Rosel, D. Trautmann, and R. Shyan, Phys. Rep. 111, 333 (1984).

12. H. Rebel and D. K. Strivastava, Preprint KfK 4761, 1990.

13. C. A. Bertulani and M. S. Hussein, Nucl. Phys. A 524, 306 (1991).

14. S. Typel and G. Baur, Phys. Rev. C 50, 2104 (1994).

15. N. Heide et al., Nucl. Phys. A 504, 374 (1989).

16. M. Yahiro, Y. Iseri, M. Kamimura, and M. Nakano, Phys. Lett. B 141, 19 (1984).

17. J. Mortimer, I. J. Thompson and J. A. Tostevin, Phys. Rev. C 65, 064619 (2002).

18. T. Matsumoto et al., Phys. Rev. C 68, 064607 (2003) (nucl-th/0302034).

19. J. W. Norbury and G. Baur, Phys. Rev. C 48, 1915 (1993).

20. H. Esbensen, G. F. Bertsch, and C. A. Bertulani, Nucl. Phys. A 581, 107 (1995).

21. C. A. Bertulani, Nucl. Phys. A 587, 318 (1995).

22. C. A. Bertulani, Z. Phys. A 356, 293 (1996).

23. S. Typel and G. Baur, Phys. Rev. C 64, 024601 (2001).

24. G. Bertsch and C. Bertulani, Phys. Rev. C 49, 2839 (1994).

25. T. Kido, K. Yabana, and Y. Suzuki, Phys. Rev. C 53, 2296 (1996).

26. V. S. Melezhik and D. Baye, Phys. Rev. C 59, 3232 (1999).

27. H. Utsunomiya et al., Nucl. Phys. A 654, 928c (1999).

28. H. Esbensen, G. F. Bertsch and K. A. Snover, Phys. Rev. Lett. 94, 042502 (2005).

29. H. D. Marta et al., Phys. Rev. C 66, 024605 (2002).

30. C. A. Bertulani, C. M. Campbell, and T. Glasmacher, Comp. Phys. Comm. 152, 317 (2003) (nucl-th/0207035).

31. P. Banerjee et al., Phys. Rev. C 65, 064602 (2002).

32. P. Banerjee and R. Shyam, Nucl. Phys. A561, 112 (1993).

33. J. A. Tostevin, F. M. Nunes, and I. J. Thompson, Phys. Rev. C 63, 024617 (2001).

34. G. Baur and H. Rebel, Ann. Rev. Nucl. Part. Sci. 46, 321 (1996).

35. J. Hesselbarth and T. K. Knöpfle, Phys. Rev. Lett. 67, 2773 (1991).

36. C. R. Brune, R. W. Kawanagh and C. Rolfs, Phys. Rev. C 50, 2205 (1994).

37. A. Shotter et al., Phys. Rev. Lett. 53, 1539 (1984) .

38. S. B. Gazes et al., Phys. Rev. Lett. 68, 150 (1992).

39. Y. Tokimoto et al., Phys. Rev. C 63, 035801 (2001) .

40. P. D. Zecher et al., Phys. Rev. C 57, 959 (1998).

41. F.-K. Thielemann, et al., Prog. Part. Nucl. Phys. 46, 5 (2001) (astro-ph/0101476)

42. A. Horvath et al., Astrophys. J. 570, 926 (2002).

43. T. Motobayashi et al., Phys. Lett. B 264, 259 (1991).

44. M. Serata et al., RIKEN Accel. Prog. Rep. 35, 62 (2002).

45. J. Kiener et al., Nucl. Phys. A 552, 66 (1993).

46. T. Motobayashi et al., Eur. Phys. J. A 13, 207 (2002).

47. T. Minemura et al., RIKEN Accel. Prog. Rep. 35, 58 (2002).

48. T. Gomi et al., RIKEN Accel. Prog. Rep. 35, 69 (2002). 
49. S. Fukuda et al., Phys. Lett. B 539, 179 (2002).

50. Q. Ahmad et al., Phys. Rev. Lett. 87, 071301 (2001).

51. P. Aliani et al., Nucl. Phys. B 634, 393 (2002).

52. J. N. Bahcall, M. N. Pinsonneault, and S. Basu, Astrophys. J. 555, 990 (2001).

53. T. Motobayashi et al., Phys. Rev. Lett. 73, 2680 (1994).

54. T. Kikuchi et al., Eur. Phys. J. A3, 213 (1998).

55. N Isawa et al., Phys. Rev. Lett. 83, 2910 (1999).

56. E. O. Alt and A. M. Mukhamedzhanov, Phys. Rev. A 47, 2004 (1993).

57. P. J. Redmond (unpublished), cited in L. Rosenberg, Phys. Rev. D 8, 1833 (1973).

58. S. P. Merkuriev, Theor. Math. Phys. 32, 680 (1977).

59. A. M. Mukhamedzhanov and M. Lieber, Phys. Rev. A 54, 3078 (1996).

60. S. B. Igamov, B. F. Irgaziev, and R. Yarmukhamedov, Phys. Atomic Nucl. 60, 2012 (1997).

61. N. A. Burkova, K. A. Zhassibekova, M. A Zhusupov, and R. A. Eramzhyan, Phys. Lett. B 248, 15 (1990).

62. A. M. Mukhamedzhanov, R. P. Schmitt, R. E. Tribble, and A. I. Sattarov, Phys. Rev. C 52, 3483 (1995).

63. G. Baur and H. Rebel, Nucl. Phys. A504, 352 (1989).

64. E. O. Alt, B. F. Irgaziev, and A. M. Mukhamedzhanov, Phys. Rev. Lett. 90, 122701 (2003).

65. E. O. Alt, B. F. Irgaziev, and A. M. Mukhamedzhanov, Phys. Rev. C 71, 024605 (2005). 